\title{
miR-24-p53 pathway evoked by oxidative stress promotes lens epithelial cell apoptosis in age-related cataracts
}

\author{
BO LU ${ }^{1}$, IAN T. CHRISTENSEN ${ }^{2}$, LI-WEI MA ${ }^{1}$, XIN-LING WANG $^{1}$, LING-FENG JIANG $^{1}$, \\ CHUN-XIA WANG ${ }^{1}$, LI FENG $^{1}$, JIN-SONG ZHANG $^{1}$ and QI-CHANG YAN ${ }^{1}$ \\ ${ }^{1}$ Department of Ophthalmology, The Fourth Affiliated Hospital of China Medical University, \\ Eye Hospital of China Medical University, Key Lens Research Laboratory of Liaoning Province, Shenyang, \\ Liaoning 110005, P.R. China; ${ }^{2}$ The School of Medicine, University of Utah, Salt Lake, UT 84132, USA
}

Received October 22, 2017; Accepted January 16, 2018

DOI: $10.3892 / \mathrm{mmr} .2018 .8492$

\begin{abstract}
MicroRNA-24 (miR-24) serves an important role in cell proliferation, migration and inflammation in various types of disease. In the present study, the biological function and molecular mechanism of miR-24 was investigated in association with the progression of age-associated cataracts. To the best of our knowledge the present study is the first to report that the expression of miR-24 was significantly increased in human anterior lens capsules affected by age-associated cataracts as well as lens epithelial cells (LECs) exposed to oxidative stress. Overexpression of miR-24 induced p53 expression and p53 was verified as a direct target of miR-24. Overexpression of miR-24 enhanced LEC death by directly targeting p53. The present study revealed that oxidative stress induced the upregulation of miR-24 and enhanced LEC death by directly targeting p53. These results suggest that the miR-24-p53 signaling pathway is involved in a novel mechanism of age-associated cataractogenesis and miR-24 may be a useful therapeutic target for age-associated cataracts.
\end{abstract}

\section{Introduction}

Cataracts represent about $42 \%$ of all causes of blindness (1) and continue to be the principal cause of blindness worldwide (2). Age-related cataracts are the most common form in adults, and are associated with visual and cognitive impairment as well as depression (3). Decreased visual function resulting from cataracts may also be responsible for a high odds ratio of nursing home placement (4) and a higher risk of mortality (5). There is a great deal of evidence suggesting

Correspondence to: Professor Qi-Chang Yan, Department of Ophthalmology, The Fourth Affiliated Hospital of China Medical University, Eye Hospital of China Medical University, Key Lens Research Laboratory of Liaoning Province, 11 Xinhua Road, Shenyang, Liaoning 110005, P.R. China

E-mail: cmu4h_yqc@126.com

Key words: miR-24, p53, oxidative stress, cataract, apoptosis that opacity of the lens in cataracts is a direct result of oxidative stress (6); however, the exact molecular mechanism of cataractogenesis remains unclear.

MicroRNAs (miRNAs) are small non-coding RNAs consisting of 19-24 nucleotides, which post-transcriptionally regulate the expression of target genes (7). Previous studies have shown that miRNAs are essential for the development of the eye and ocular homeostasis (8), and miRNA dysregulation may play an important role in ocular diseases including cataracts $(9,10)$. In addition, a number of miRNAs have been reported as potential diagnostic biomarkers or therapeutic targets for cataracts $(11,12)$.

MicroRNA-24 (miR-24) has diverse functions in cell proliferation (13-15), and regulates key aspects of age-related macular degeneration pathology $(16,17)$. The biological function of miR-24 in the progression of cataract development is still unclear. In the present study, we found for the first time that miR-24 is more highly expressed in age-related cataracts, and enhances lens epithelial cell apoptosis by directly targeting p53. The miR-24-p53 pathway may play a critical role in cataractogenesis.

\section{Materials and methods}

Specimens. The present study was approved by the Ethical Committee of the Fourth Affiliated Hospital of China Medical University. Written informed consent was obtained from each patient. Age-related cataract patients without other ocular diseases undergoing cataract surgery (phacoemulsifcation) were enrolled at the Fourth Affiliated Hospital of China Medical University (Shenyang, China). Normal eyes were obtained from the Eye Bank of the Fourth Affiliated Hospital of China Medical University. Fresh anterior lens capsules isolated from age-related cataract patients and normal eyes were immediately frozen in liquid nitrogen at the time of surgery and stored at $-80^{\circ} \mathrm{C}$.

Cell culture. Human lens epithelial cell line cells (SRA01/04 cells, a kind gift of Dr. Yi-sin Liu, Doheny Eye Institute, Los Angeles, CA, USA) were maintained in Dulbecco's modified Eagle's medium (DMEM; Gibco; Thermo Fisher Scientific, Inc., Waltham, MA, USA), supplemented with $10 \%$ fetal bovine serum 
(FBS; Gibco; Thermo Fisher Scientific, Inc.), $100 \mathrm{U} / \mathrm{ml}$ penicillin and $100 \mathrm{mg} / \mathrm{ml}$ streptomycin (Thermo Fisher Scientific, Inc.) in a humidifed incubator at $37^{\circ} \mathrm{C}$ with $5 \% \mathrm{CO}_{2}$.

Reverse transcription-quantitative polymerase chain reaction $(R T-q P C R)$. Total RNA was extracted from tissues and cells using the Trizol Reagent (Invitrogen; Thermo Fisher Scientific, Inc.) according to the manufacturer's instructions. For RT-qPCR analysis of miR-24, the total RNA isolated from cells was subsequently reverse transcribed to cDNA using a TaqMan MicroRNA Reverse Transcription Kit (Applied Biosystems; Thermo Fisher Scientific, Inc.). The expression of miR-24 was determined using TaqMan MicroRNA assays (Applied Biosystems; Thermo Fisher Scientific, Inc.), and standardized to RNU6B expression. The upstream and downstream primers of miR-24 and RNU6B were purchased from Thermo Fisher Scientific, Inc., and their sequences can be found on their website. For RT-qPCR analysis of p53, cDNAs were obtained using the PrimerScript RT reagent kit (Takara Bio Inc., Tokyo, Japan), RT-qPCR analysis of p53 was performed with TaqMan Universal Master Mix II (Applied Biosystems; Thermo Fisher Scientific, Inc.). Primers for p53 were as follows: Forward 5'-CAGCAGTCA AGCACTGCC AAG-3', reverse 5'-AGACAGGCATGGCACGGATAA-3', and $\beta$-actin was used for normalization, $\beta$-actin primer sequences were: Forward: 5'-CATCCGTAAAGACCTCTATGCCAA C-3', Reverse: 5'-ATGGAGCCACCGATCCACA-3'. The RT-qPCR analysis was performed on ABI 7500 (Applied Biosystems; Thermo Fisher Scientific, Inc.). All experiments were performed in triplicate. The relative expression levels of mRNA or microRNA were calculated using the $2^{-\Delta \Delta C t}$ method.

Western blot analysis. Total protein was extracted using a RIPA lysis buffer supplemented with a protease inhibitor cocktail (Pierce; Thermo Fisher Scientific, Inc.). Equal amounts (40 $\mu \mathrm{g}$ ) of proteins were separated using NuPAGE 4-12\% Bis-Tris Protein gels (Invitrogen; Thermo Fisher Scientific, Inc.), then transferred to PVDF membranes (EMD Millipore, Billerica, MA, USA). The membranes were subsequently blocked with $5 \%$ fat-free milk at room temperature for $2 \mathrm{~h}$ and incubated with the primary antibodies including rabbit anti-p53 (1:1,000; Abcam, Cambridge, CA, USA) and rabbit anti-GAPDH (1:2,000, Santa Cruz Biotechnology, Inc., Dallas, TX, USA) at $4^{\circ} \mathrm{C}$ overnight, followed by incubation with horseradish peroxidase (HRP)-conjugated goat anti-rabbit $\operatorname{IgG}(\mathrm{H}+\mathrm{L})$ secondary antibody (1:2,500; Promega Corporation, Madison, WI, USA) at room temperature for $2 \mathrm{~h}$. The protein bands were visualized using the an ECL Western Blotting Substrate kit (Pierce; Thermo Fisher Scientific, Inc.) and quantified using Image J software (National Institute of Health, USA).

Detection of reactive oxygen species (ROS) level. A $2^{\prime}, 7^{\prime}$-dichloro-fluorescein diacetate (DCFH-DA) probe was used to detect fluorescence derived from endogenous ROS in hLECs. $1 \times 10^{4}$ cells were seeded into each well of a 96-well plate and were cultured for $16 \mathrm{~h}$, until cells were observed adhering to the sides of the well. The cells were then exposed to different concentrations $\mathrm{H}_{2} \mathrm{O}_{2}(0,100,200,400,600,800$, $1,000 \mu \mathrm{M})$ for $1 \mathrm{~h}$ whereupon the culture medium was aspirated and $10 \mathrm{uM}$ fluorescent probe DCFH-DA was added to each well. This mixture was then incubated in a $37^{\circ} \mathrm{C}$ incubator for $20 \mathrm{~min}$. The cells were then washed three times with PBS and their DCF fluorescence intensity value (i.e., the mean fluorescence intensity of DCF, representing the level of intracellular ROS) was read using a multifunctional microplate reader. The excitation wavelength used was $485 \mathrm{~nm}$ and the emission wavelength was set at $530 \mathrm{~nm}$.

Cell viability assay. Cell viability and proliferation were determined using the CellTiter96AQ ${ }_{\text {ueous }}$ One Solution Cell Proliferation assay kit (Promega). The reagent contains a tetrazolium compound (3-(4,5-dimethylthiazol-2-yl)-5(3-carboxymethoxyphenyl)-2-(4-sulfophenyl)-2H-tetrazolium, inner salt; MTS). After treatments, according to the manufacturer's protocols, $20 \mu \mathrm{l}$ MTS solution was added to each well of the 96-well assay plate containing the cells in $100 \mu 1$ of culture medium and the cells were then incubated for $4 \mathrm{~h}$ at $37^{\circ} \mathrm{C}, 5 \% \mathrm{CO}_{2}$. The absorbance of each group was read using an absorbance plate reader set to a $490 \mathrm{~nm}$ wavelength. The cell viability rates were calculated according to the following formula: The cell viability ratio $(\%)=[(\mathrm{As}-\mathrm{Ab}) /(\mathrm{Ac}-\mathrm{Ab})] \times 100 \%$, where As is the optical density value at $490 \mathrm{~nm}$ (OD490) of $\mathrm{H}_{2} \mathrm{O}_{2}$ treatment group, $\mathrm{Ab}$ is the OD490 of blank group, and $\mathrm{Ac}$ is the OD490 of non- $\mathrm{H}_{2} \mathrm{O}_{2}$ control group. Each experiment was repeated three times.

Caspase- 3 activity assay. Caspase- 3 activity was detected using a caspase-3 assay kit (Abcam). After treatments, in accordance with the manufacturer instructions, these SRA01/04 cells were lysed in $50 \mu \mathrm{l}$ of chilled Cell Lysis buffer and incubated on ice for $10 \mathrm{~min}$, centrifuged, the supernatant protein concentration was determined using the BCA method. $50 \mu 1$ of Cell Lysis buffer containing $100 \mu \mathrm{g}$ protein were added to each well in a 96 well plate. Then, $50 \mu 12 \mathrm{x}$ Reaction buffer, $0.5 \mu \mathrm{l} 10 \mathrm{mM}$ DTT and $5 \mu \mathrm{l}$ caspase- 3 catalytic substrate DEVD-p-NA substrate were added to each well. The samples were incubated at $37^{\circ} \mathrm{C}$ for $2 \mathrm{~h}$. The optical density (OD) value was obtained using a microplate reader set at $405 \mathrm{~nm}$ wavelength. Each experiment was repeated three times. In the caspase- 3 activity control group, OD was set to a value of 1 and the caspase- 3 experimental group activity was standardized using the following calculation: (OD value of experimental group-blank well OD)/(OD value of control group-blank well OD) x100\%.

Transient transfection. The miR-24 mimic (miR-24), mimic negative control (miR-Ctrl), miR-24 inhibitor (anti-miR-24), inhibitor negative control (anti-miR-Ctrl), small interfering RNA for $\mathrm{p} 53$ (p53 siRNA) and siRNA control were purchased from GenePharma, Inc., (Sunnyvale, CA, USA). SRA01/04 cells were seeded in a 6-well plate, and transfection was conducted after $24 \mathrm{~h}$. Transfections were performed according to the manufacturer's instructions with Lipofectamine RNAiMAX Transfection Reagent (Invitrogen; Thermo Fisher Scientific, Inc.). After $72 \mathrm{~h}$, the cells were treated with $400 \mu \mathrm{M} \mathrm{H}_{2} \mathrm{O}_{2}$ for $1 \mathrm{~h}$ after which the expression of miR-24 was measured using RT-qPCR. The expression of p53 was measured using RT-qPCR and western blotting, and the cell viability was measured using MTS.

Luciferase reporter assay. We used human cDNA to generate wild-type and mutant 3'-UTR sequences for the p53 gene, 
including predicted miR-24 targeting regions. We then cloned these amplified fragments into the pGL3-Promoter vector (Promega) at the same location. For luciferase reporter assays, these reporters were cotransfected into SRA01/04 cells together with miR-24 mimics and mimic controls. Luciferase activity was then evaluated using a Dual-Luciferase Reporter Assay System kit (Promega) at $72 \mathrm{~h}$ after transfection. The Renilla luciferase plasmid was used as an endogenous control. The experiments were performed in triplicate.

Statistical analysis. Each experiment was repeated independently at least 3 times with similar results. Measurement data were presented as mean \pm standard deviation (SD). Differences between two groups were calculated using an independent sample t-test. Differences among multiple groups were determined by one-way analysis of variance followed by Dunnett's post hoc test. $\mathrm{P}<0.05$ was considered to indicate a statistically significant difference. Statistical analysis was done using SPSS v16.0 (SPSS, Inc., Chicago, IL, USA).

\section{Results}

Increased expression of miR-24 in the anterior lens capsules of patients with age-related cataracts. One previously conducted microarray study observed that miR-24 levels dramatically changed in human cataractous lenses (18), but this finding has not been verified. To explore the expression levels of miR-24 in the lens epithelial cells (LECs) of age-related cataracts, RNAs isolated from the 48 anterior lens capsules of age-related cataract patients and the 32 normal anterior lens capsule specimens were subjected to RT-qPCR analysis. The assays showed that the expression of miR-24 was significantly increased in cataract tissues as compared to normal tissues (Fig. 1). These results suggest that miR-24 may play a role in cataract development.

p53 is up-regulated in the anterior lens capsules of patients with age-related cataracts. p53 has been implicated as an important protein in cell proliferation and differentiation during embryonic lens development (19). In the present study, the expression of p53 was also examined by RT-qPCR and western blotting in anterior lens capsules (control: $n=44$, cataract: $n=56$ ). Both the expression of $\mathrm{p} 53$ protein and $\mathrm{p} 53$ mRNA were found to be significantly up-regulated in the cataract tissues when compared with the normal tissues (Fig. 2). This finding, combined with the previous miR-24 experiment (Fig. 1) demonstrates a positive correlation between endogenous miR-24 and p53 expression.

Increased levels of miR-24 and p53, enhanced expression of ROS, and increased cell death and cell apoptosis were detected in LECs exposed to oxidative stress induced by $\mathrm{H}_{2} \mathrm{O}_{2}$. In order to establish the expression levels of miR-24 and p53 in LECs exposed to oxidative stress, we treated SRA01/04 cells with $400 \mu \mathrm{mol} \mathrm{H}_{2} \mathrm{O}_{2}$ for $1 \mathrm{~h}$. The expression of miR-24 was detected using RT-qPCR which showed a great increase in SRA01/04 cells exposed to $\mathrm{H}_{2} \mathrm{O}_{2}$ compared with controls (Fig. 3A). RT-qPCR (Fig. 3B) and western blotting analysis of p53 (Fig. 3C and D) revealed a significant increase of p53 in SRA01/04 cells treated with $\mathrm{H}_{2} \mathrm{O}_{2}$. This increase

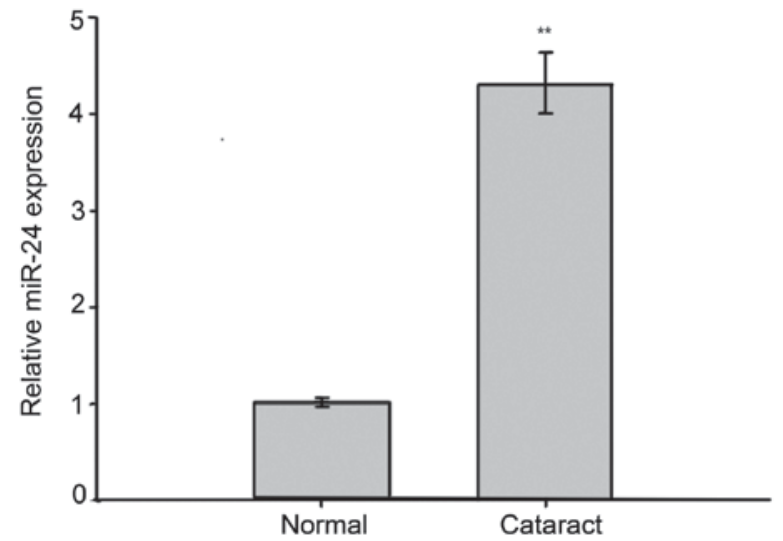

Figure 1. The expression of miR-24 was significantly increased in age-related cataract tissues compared to normal tissues. The relative levels of miR-24 expression in 48 specimens of anterior lens capsule of age-related cataract and 32 normal anterior lens capsule specimens were measured by RT-qPCR analysis. Data are representative of three independent experiments. Values are presented as mean $\pm \mathrm{SD}$. SD, standard deviation; ${ }^{* *} \mathrm{P}<0.01$ compared to normal groups.

was correlated with higher expression of ROS (Fig. 3E), decreased cell viability (Fig. 3F) and increased caspase-3 activity (Fig. 3G). This suggests that ROS modulated both miR-24 and p53 in LECs.

miR-24 regulated the expression of p53 protein and $m R N A$ in LECs exposed to oxidative stress. To investigate the correlation between miR-24 and p53 expression in LECs exposed to oxidative stress, we transfected SRA01/04 cells with either miR-24 mimics or inhibitors, then the medium was removed and $400 \mu \mathrm{mol} \mathrm{H}_{2} \mathrm{O}_{2}$ was added to induce oxidative stress. RT-qPCR was performed to assess the level of p53-mRNA expression. As is shown in Fig. 4A and $\mathrm{B}$, samples with overexpressed miR-24 up-regulated p53 mRNA expression when compared with controls, while inhibition of miR-24 down-regulated the expression of p53 mRNA. Western blotting indicated that p53 protein levels were enhanced in SRA01/04 cells transfected with miR-24 mimics and reduced in cells exposed to miR-24 inhibitors (Fig. 4C-F). These results indicated that the expression of p53 was regulated at both the mRNA and protein level by miR-24 in LECs.

p53 was verified as a direct target of $m i R-24$. To more closely examine the mechanisms of miR-24 and p53 in cataracts, we used bioinformatics with publicly available databases (TargetScan, miRanda and miRBase) to determine whether p53 may be the target of miR-24 (Fig. 5A). To confirm the targeting of p53 by miR-24, luciferase activity assays were performed. SRA01/04 cells were co-transfected the luciferase reporter construct pGL3-p53-wt or pGL3-p53-mut with miR-24 mimics. As shown in Fig. 5B, SRA01/04 cells with pGL3-p53-wt and miR-24mimics had significantly increased reporter activity when compared with the controls, whereas no significant difference in reporter activity was observed when the target site was mutated. Together, these results indicate that 3'UTR of p53 carries a direct and functional binding site for miR-24 in LECs. 


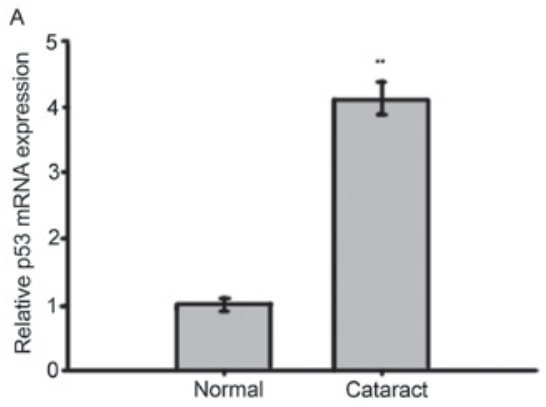

B

p53

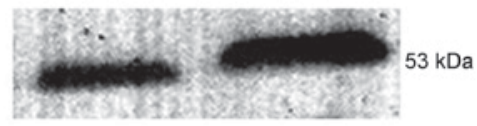

GAPDH
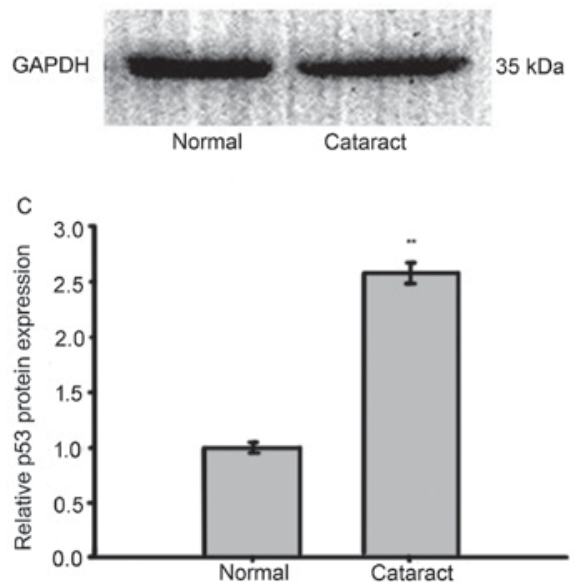

Figure 2. p53 is up-regulated in the anterior lens capsule of patients with age-related cataracts. Both the expression of (A) p53 mRNA and (B) p53 protein were found to be significantly up-regulated in the cataract tissues when compared with the normal tissues. (C) Strip chart of p53 protein. Data are representative of three independent experiments. Values are presented as mean $\pm \mathrm{SD}$. SD, standard deviation; ${ }^{* *} \mathrm{P}<0.01$ compared to normal groups.
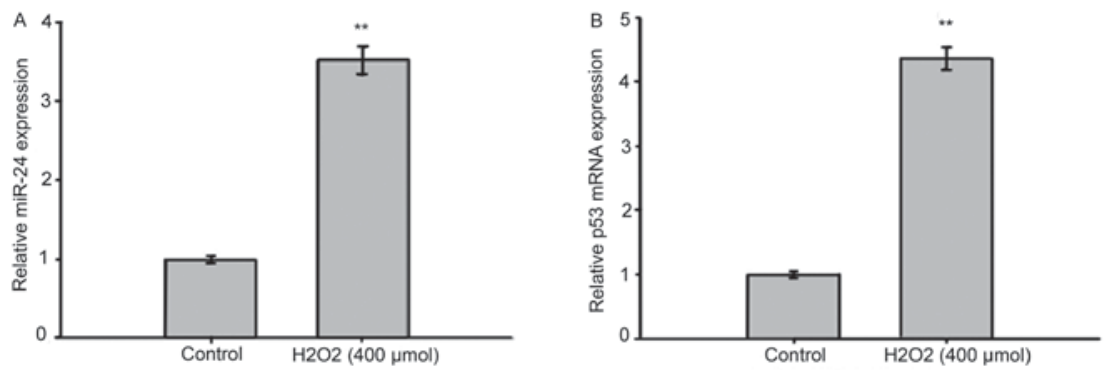

c

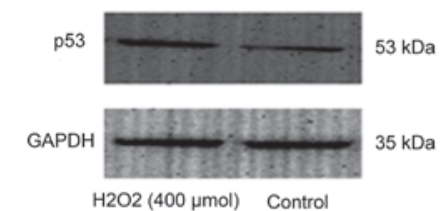

$\mathrm{E}$
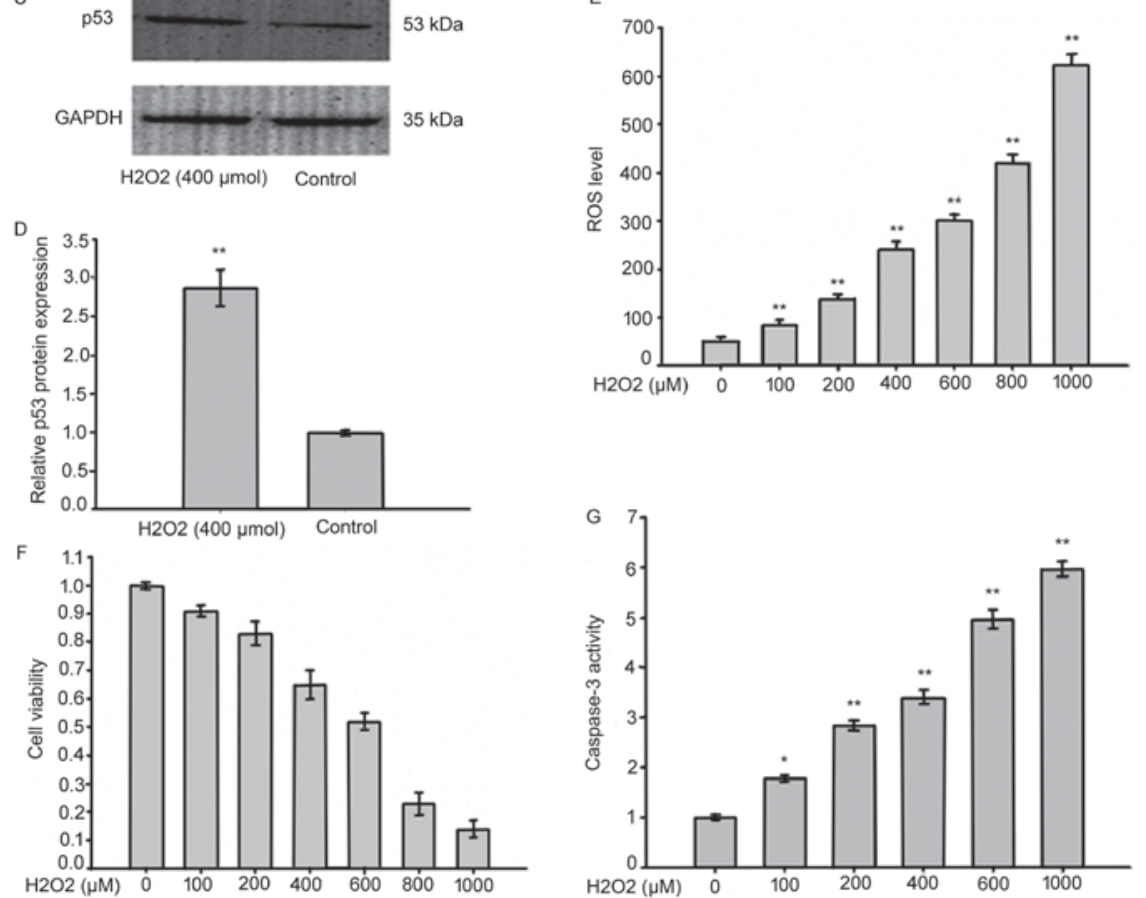

Figure 3. Increased levels of miR-24 and p53 with enhanced expression of ROS and cell death were detected in LECs exposed to oxidative stress induced by $\mathrm{H}_{2} \mathrm{O}_{2}$. SRA01/04 cells were treated with $400 \mu \mathrm{mol} \mathrm{H} \mathrm{O}_{2}$ for $1 \mathrm{~h}$. (A) The expression of miR-24 was detected using RT-qPCR. (B) The expression of p53 mRNA was detected using RT-qPCR. (C) The expression of p53 protein was detected using western blotting. (D) Strip chart of p53 protein. (E) SRA01/04 cells were treated with different concentrations of $\mathrm{H}_{2} \mathrm{O}_{2}$ for $1 \mathrm{~h}$. Intracellular ROS levels were monitored. (F) MTS assay was performed to monitor cell viability against oxidative stress. (G) Caspase-3 activity assay was performed to monitor cell caspase-3 activity against oxidative stress. Data are representative of three independent experiments. Values are presented as mean $\pm \mathrm{SD}$. SD, standard deviation; ${ }^{*} \mathrm{P}<0.05,{ }^{* *} \mathrm{P}<0.01$ compared to controls. 

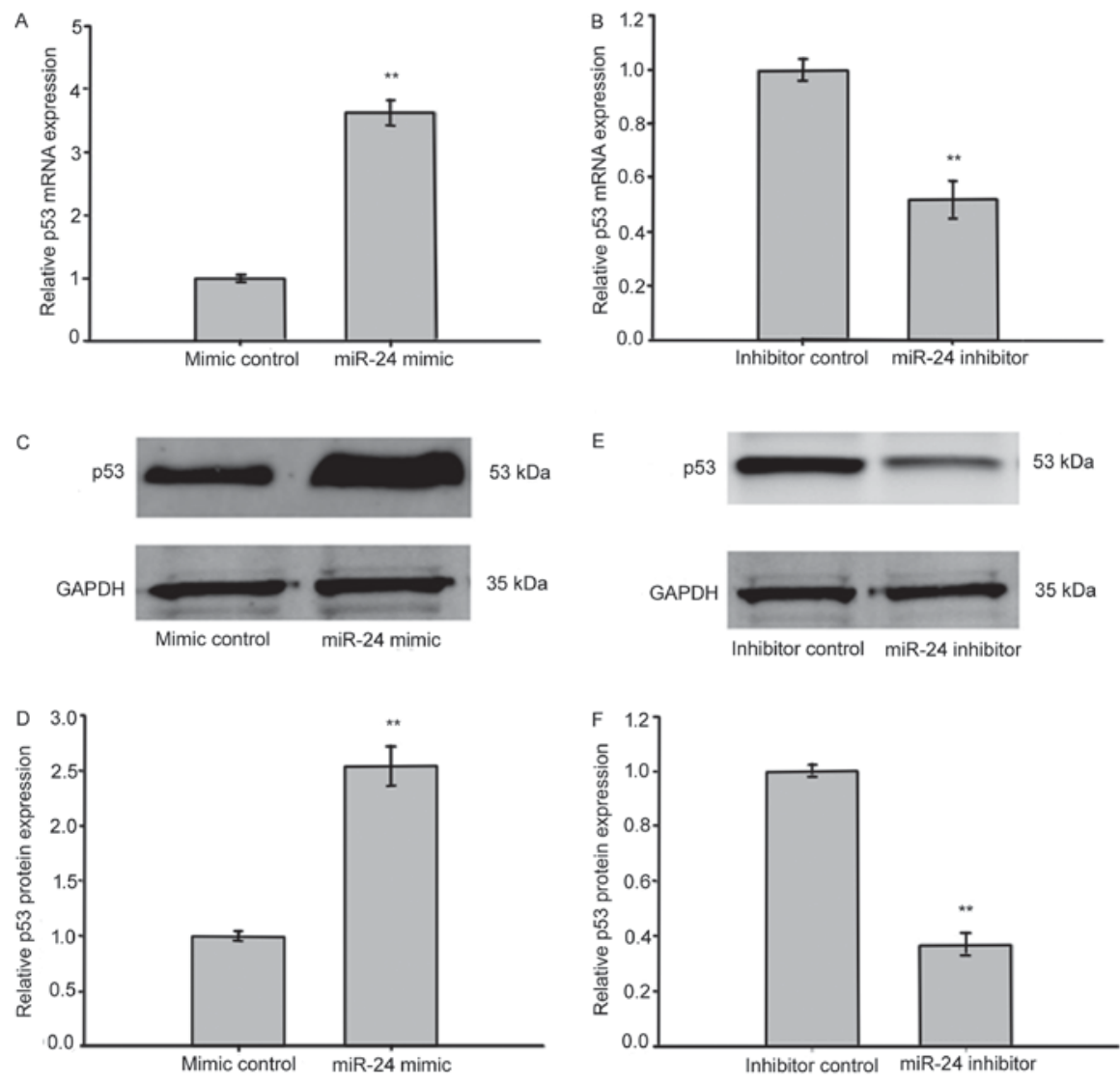

Figure 4. miR-24 regulated the expression of p53 protein and mRNA in LECs exposed to oxidative stress. We transfected SRA01/04 cells with either miR-24 mimics or inhibitors, then the medium was removed and $400 \mu \mathrm{mol} \mathrm{H}_{2} \mathrm{O}_{2}$ was added to induce oxidative stress. (A) Relative level of p53 mRNA expression in LECs transfected with miR-24 mimics. (B) Relative level of p53 mRNA expression in LECs transfected with miR-24 inhibitors. (C) Western blotting indicated that $\mathrm{p} 53$ protein levels were enhanced in SRA01/04 cells transfected with miR-24 mimics. (D) Strip chart of p53 protein. (E) p53 protein levels were reduced in cells exposed to miR-24 inhibitors. (F) Strip chart of p53 protein. Data are representative of three independent experiments. Values are presented as mean $\pm \mathrm{SD}$. SD. standard deviation; ${ }^{* *} \mathrm{P}<0.01$ compared to control.

A

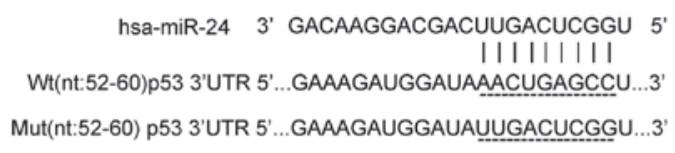

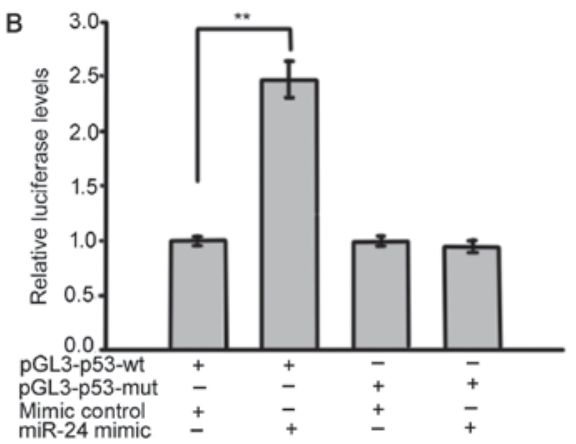

Figure 5. p53 was verified as a direct target of miR-24. (A) Bioinformatic analysis of the wild-type and mutant miR-24 target sequences with the 3'UTR of p53 cDNA. (B) Relative luciferase activity evaluated by Dual-Luciferase Reporter Assay. The luciferase activity of SRA01/04 cells with pGL3-p53-wt and miR-24 mimics increased nearly 2 times compared with the controls. Data are representative of three independent experiments. Values are presented as mean \pm SD. $\mathrm{SD}$, standard deviation; ${ }^{* *} \mathrm{P}<0.01$ compared to control.

miR-24 enhanced LEC death and apoptosis induced by oxidative stress. To identify the role of miR-24 in LEC viability and apoptosis, we used MTS assays to measure the viability of LECs, and caspase-3 activity was also assessed. The SRA01/04 cells were transfected with miR-24 mimics, mimic controls, miR-24 inhibitors and inhibitor controls before exposure to $\mathrm{H}_{2} \mathrm{O}_{2}(400 \mu \mathrm{mol})$. As assayed by MTS, SRA01/04 cells transfected with miR-24 mimics displayed significantly increased cell death compared to those transfected with mimic controls, while transfection with miR-24 inhibitors significantly suppressed $\mathrm{H}_{2} \mathrm{O}_{2}$-induced LEC death (Fig. 6A). Results of caspase-3 activity shown that compared with the control group, the miR-24 mimic group had significantly elevated caspase- 3 activity while the caspase- 3 activity of the miR-24 inhibitor group was markedly decreased (Fig. 6B). These results suggest that miR-24 promotes apoptosis and inhibits the proliferation of human LECs exposed to oxidative stress. 

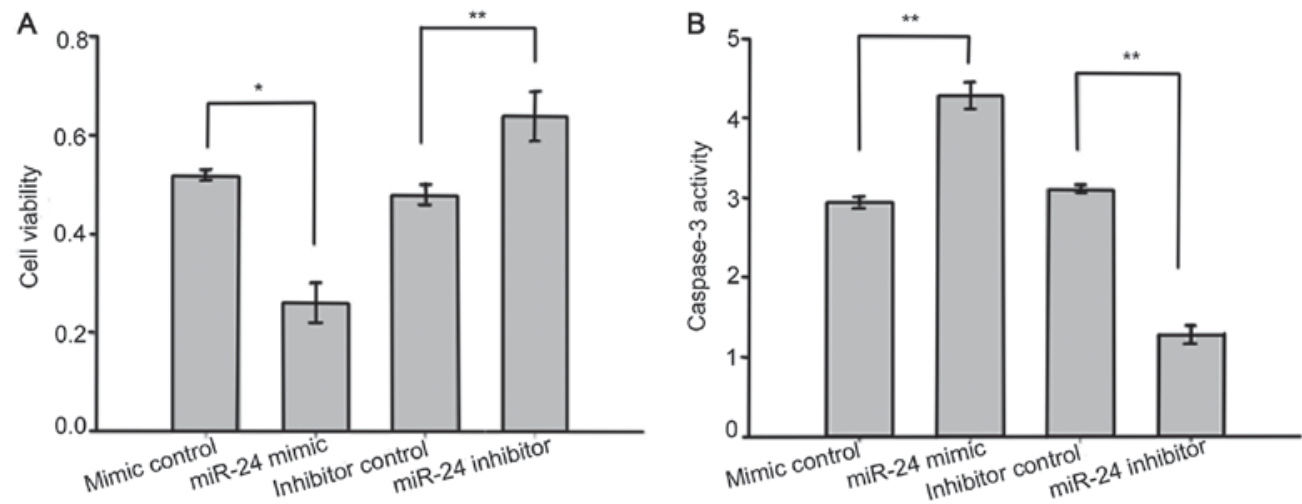

Figure 6. miR-24 enhanced LEC death and apoptosis induced by oxidative stress. (A) The viability of LECs measured by MTS assays. (B) The caspase-3 activity of LECs measured by caspase-3 activity assays. Data are representative of three independent experiments. Values are presented as mean \pm SD. SD. standard deviation; ${ }^{*} \mathrm{P}<0.05,{ }^{* *} \mathrm{P}<0.01$ compared to control.

A p53 (1)

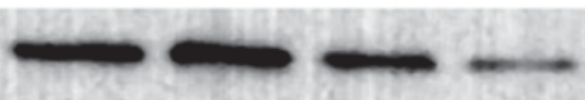

$53 \mathrm{kDa}$

\section{GAPDH}

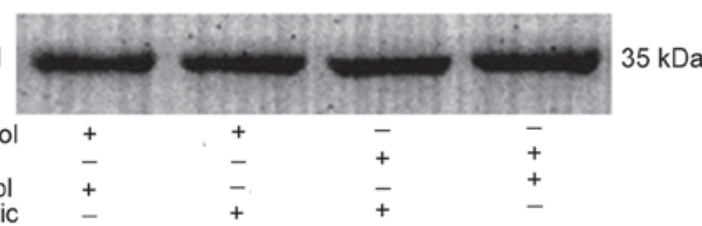

B

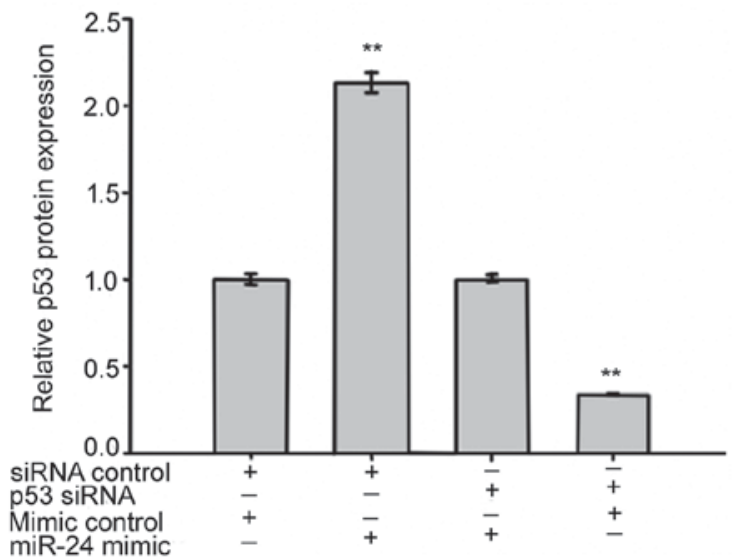

C

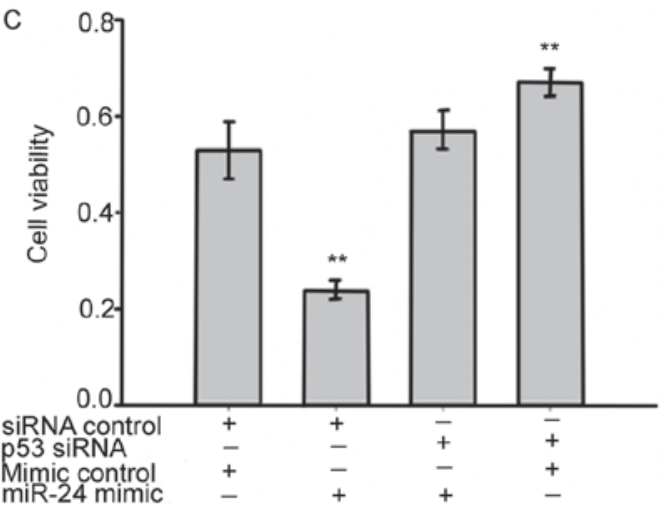

D

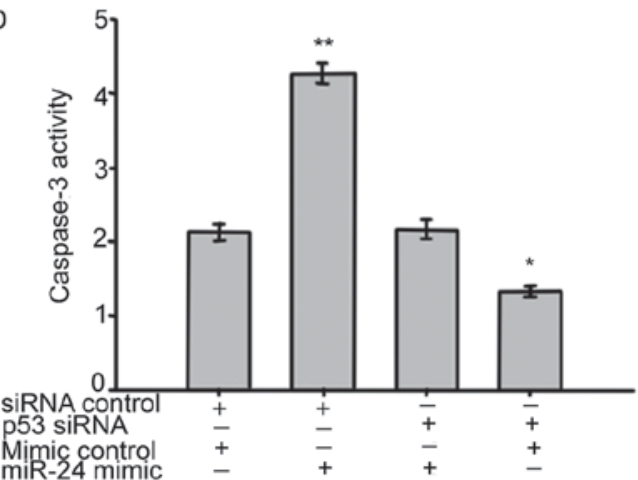

Figure 7. miR-24 enhanced LEC death and apoptosis by targeting p53. Before exposure to $\mathrm{H}_{2} \mathrm{O}_{2}(400 \mu \mathrm{mol})$, SRA01/04 cells were co-transfected with p53 siRNA in the absence or presence of miR-24 mimics. $72 \mathrm{~h}$ after transfection, the protein expression of p53 was measured by western blotting (A) and (B), (C) Cell viability assessed by MTS assays. (D) The caspase-3 activity measured by caspase-3 activity assays. Data are representative of three independent experiments. Values are presented as mean $\pm \mathrm{SD}$. SD, standard deviation; $\mathrm{P}<0.05,{ }^{* *} \mathrm{P}<0.01$ compared to control.

miR-24 enhanced LEC death by targeting p53. The previous experiments clearly indicated that miR-24 enhances LEC death. To explore whether p53 is involved in miR-24 enhanced LEC death and apoptosis, we knocked down p53 using p53 siRNA. Before exposure to $\mathrm{H}_{2} \mathrm{O}_{2}$, SRA01/04 cells were transfected with p53 siRNA in the absence or presence of miR-24 mimics. $72 \mathrm{~h}$ after transfection, cell viability was assessed by MTS assays, caspase- 3 activity was assessed by caspase- 3 activity assays and the protein expression of p53 was measured by western blotting. Co-transfection of miR-24 mimics with p53 siRNA significantly reversed the p53 protein expression induced by miR-24 mimics transfection (Fig. 7A and B). As shown in
Fig. 7C and D, when compared with cells co-transfected with the mimic controls and the siRNA controls, the viability and caspase-3 activity of LECs co-transfected with miR-24 mimics and p53 siRNA showed little change. In addition, knockdown of p53 in LECs resulted in an increased cell survival rate and decreased caspase- 3 activity. These results indicate that miR-24 enhanced LEC death and apoptosis by directly targeting p53.

\section{Discussion}

The major unique finding in this study is that the oxidative stress induced upregulation of miR-24 enhances LEC apoptosis 
and inhibits LEC proliferation by directly targeting p53. Our experimental design elucidated the molecular mechanism of p53 regulation by miR-24 under oxidative stress. To the best of our knowledge, this experiment is also the first to report that the expression of miR-24 was significantly increased in human anterior lens capsules affected by age-related cataracts as well as LECs exposed to oxidative stress. Our data also showed that miR-24 expression was positively associated with p53 levels. These data suggest that the miR-24-p53 pathway is involved in a novel mechanism of age-related cataractogenesis.

There is a growing body of evidence that indicates miRNAs play an important role in the development of the eye, ocular homeostasis, and ocular diseases (20). In previously published research, miRNA expression profiles in human lenses were identified through the use of microarrays. The top eight miRNAs in cataractous lenses were miR-184, miR-1826, let-7b/c, miR-24, miR-23b, miR-923, and miR-23a (18). Our research further determined the miR-24 levels in the anterior lens capsule of patients with age-related cataracts, and for the first time discovered that miR-24 expression was significantly upregulated in lenses with age-related cataracts as compared to transparent lenses. This fact suggests that the differential expression of miR-24 may play a role in cataractogenesis.

It has been widely reported that the lens is subjected to oxidative stress throughout its life and that oxidative damage is a major cause of cataract formation (21). Current research also suggests that with increasing age, the accumulation of oxidized lens components and decreased capacity of repair mechanisms result in increased levels of reactive oxidative species (22) and LEC death, promoting cataractogenesis $(21,23)$. In the present study, we used $\mathrm{H}_{2} \mathrm{O}_{2}$ to induce LEC death and apoptosis as an oxidative stress model.

The p53 signaling pathway plays an important role in regulating the cell cycle and cell differentiation, promoting apoptosis, and activating cell death (24-26). It has been previously reported that LECs exposed to $\mathrm{H}_{2} \mathrm{O}_{2}$-induced oxidative stress have increased expression of p53 protein $(27,28)$. In addition, LECs with diabetic cataracts (29) and age-related cataracts (11) were also found to have higher levels of p53 expression. Chen et al (13), found that miR-24 increases hepatocellular carcinoma cell metastasis and invasion by targeting p53. This study showed a positive correlation between endogenous miR-24 and p53 expression in the anterior lens capsule of patients with age-related cataracts. Consequently, it seems likely that miR-24 enhances human LEC apoptosis through the activation of p53.

By using a human lens epithelial cell line (SRA01/04 cells) as an in vitro model to study the effects of aging and oxidative stress, we determined that the levels of both miR-24 and p53 were elevated and linked this heightened expression with increased levels of ROS. We were then able to demonstrate that ROS promote the miR-24-p53 pathway. The key novel observation of this study is that miR-24 directly targeted p53 in human LECs, promoting cell apoptosis and inhibiting cell proliferation. Taken together, these findings indicate that the miR-24 evoked by oxidative stress enhances LEC apoptosis and inhibits LEC proliferation by directly targeting p53, also contributing to the development of cataracts.

In recent years, miRNAs have emerged as one of the most reliable diagnostic biomarkers and therapeutic targets in a variety of diseases $(29,30)$. miRNA-based therapeutics involve modulating the functions of disease associated miRNAs by miRNA antagonists or mimics (31-33). For example, Miravirsen which is a $\beta$-D-oxy-locked nucleic acid-modified phosphorothioate antisense oligonucleotide targeting the liver-specific miR-122 has demonstrated broad antiviral activity and a relatively high genetic barrier to resistance in clinical trial study $(34,35)$.

Although miRNA-based diagnostic tools and therapeutics for ocular diseases are still on the horizon, there have been several studies in recent years to suggest their potential for clinical use. For example, Li et al (36) established miR-143 and miR-145 as important regulators of intraocular pressure, which may have important therapeutic implications in glaucoma. Additionally, overexpressing miR-21, miR-31, miR-150, and miR-146a, or silencing miR-23/27, have each been suggested as potential approaches for treating choroidal neovascularization in wet age-related macular degeneration (31,37-39). Finally, miR-133b and miR-125b were shown to be downregulated in age-related cataracts and appeared to inhibit lens epithelial cell apoptosis $(11,12)$.

Current investigations into the subject of our study, miR-24, is mostly limited to cancer research. For instance, Van Eijndhoven et al (40) reported that purified extracellular vesicles fractions of untreated classical Hodgkin lymphoma patients had enriched levels of miR-24 and the concentration of miR-24 decreased during and after therapy, suggesting miR-24 reflects the presence of vital tumor tissue and is suitable for therapy response and relapse monitoring in individual classical Hodgkin lymphoma patients. In other studies, miR-24 was found to suppress cell migration, invasion, and proliferation in breast cancer, osteosarcoma and nasopharyngeal carcinoma (41-44), indicating that miR-24 could be a potential target for the diagnosis and therapy of cancer.

Ophthalmology research related to miR-24, by contrast, has been less extensive. There is some evidence that overexpression of miR-24 is effective in repressing choroidal neovascularization in vivo, suggesting miR-24 may represent an attractive therapeutic solution for wet age-related macular degeneration $(16,45)$. Unfortunately, data on miR-24 in cataracts are still scarce.

In conclusion, miR-24 is up-regulated in age-related cataracts. It appears to enhance lens epithelial cell apoptosis and inhibit cell proliferation by directly targeting $\mathrm{p} 53$, suggesting that the miR-24-p53 pathway may play a critical role in cataractogenesis. These findings support the possibility of miR-24 as a desirable therapeutic target for age-related cataracts.

\section{Acknowledgements}

The present study was supported by grants from the National Natural Science Foundation of China (grant nos. 81170836, 81570838) and the Natural Science Foundation of Liaoning Province, China (grant no. 2015020474).

\section{References}

1. Goutham G, Manikandan R, Beulaja M, Thiagarajan R, Arulvasu C, Arumugam M, Setzer WN, Daglia M, Nabavi SF and Nabavi SM: A focus on resveratrol and ocular problems, especially cataract: From chemistry to medical uses and clinical relevance. Biomed Pharmacother 86: 232-241, 2017. 
2. Lee CM and Afshari NA: The global state of cataract blindness. Curr Opin Ophthalmol 28: 98-103, 2017.

3. Fukuoka $\mathrm{H}$ and Afshari NA: The impact of age-related cataract on measures of frailty in an aging global population. Curr Opin Ophthalmol 28: 93-97, 2017.

4. Aditya BS, Sharma JC, Allen SC and Vassallo M: Predictors of a nursing home placement from a non-acute geriatric hospital. Clin Rehabil 17: 108-113, 2003.

5. Khanna RC, Murthy GV, Giridhar P, Krishnaiah S, Pant HB, Palamaner Subash Shantha G, Chakrabarti S, Gilbert C and Rao GN: Cataract, visual impairment and long-term mortality in a rural cohort in India: The Andhra Pradesh Eye Disease Study. PLoS One 8: e78002, 2013.

6. Kubota M, Shui YB, Liu M, Bai F, Huang AJ, Ma N, Beebe DC and Siegfried CJ: Mitochondrial oxygen metabolism in primary human lens epithelial cells: Association with age, diabetes and glaucoma. Free Radic Biol Med 97: 513-519, 2016.

7. Ebert MS and Sharp PA: Roles for microRNAs in conferring robustness to biological processes. Cell 149: 515-524, 2012.

8. Xu S: microRNA expression in the eyes and their significance in relation to functions. Prog Retin Eye Res 28: 87-116, 2009.

9. Dunmire JJ, Lagouros E, Bouhenni RA, Jones M and Edward DP: MicroRNA in aqueous humor from patients with cataract. Exp Eye Res 108: 68-71, 2013.

10. Szemraj M, Bielecka-Kowalska A, Oszajca K, Krajewska M, Goś R, Jurowski P, Kowalski M and Szemraj J: Serum MicroRNAs as potential biomarkers of AMD. Med Sci Monit 21: 2734-2742,2015.

11. Qin Y, Zhao J, Min X, Wang M, Luo W, Wu D, Yan Q, Li J, Wu X and Zhang J: MicroRNA-125b inhibits lens epithelial cell apoptosis by targeting p53 in age-related cataract. Biochim Biophys Acta 1842: 2439-2447, 2014

12. Zhang F, Meng $W$ and Tong B: Down-regulation of MicroRNA-133b suppresses apoptosis of lens epithelial cell by up-regulating BCL2L2 in age-related cataracts. Med Sci Monit 22: 4139-4145, 2016.

13. Chen L, Luo L, Chen W, Xu HX, Chen F, Chen LZ, Zeng WT, Chen JS and Huang XH: MicroRNA-24 increases hepatocellular carcinoma cell metastasis and invasion by targeting p53: miR-24 targeted p53. Biomed Pharmacother 84: 1113-1118, 2016.

14. Chen W and Ou HS: Regulation of miR-24 on vascular endothelial cell function and its role in the development of cardiovascular disease. Sheng Li Xue Bao 68: 201-206, 2016 (In Chinese).

15. Yang J, Chen L, Ding J, Fan Z, Li S, Wu H, Zhang J, Yang C, Wang $\mathrm{H}$, Zeng $\mathrm{P}$ and Yang J: MicroRNA-24 inhibits high glucose-induced vascular smooth muscle cell proliferation and migration by targeting HMGB1. Gene 586: 268-273, 2016.

16. Ertekin S, Yildirim O, Dinc E, Ayaz L, Fidanci SB and Tamer L: Evaluation of circulating miRNAs in wet age-related macular degeneration. Mol Vis 20: 1057-1066, 2014.

17. Kutty RK, Samuel W, Jaworski C, Duncan T, Nagineni CN, Raghavachari N, Wiggert B and Redmond TM: MicroRNA expression in human retinal pigment epithelial (ARPE-19) cells: Increased expression of microRNA-9 by N-(4-hydroxyphenyl) retinamide. Mol Vis 16: 1475-1486, 2010.

18. Wu C, Lin H, Wang Q, Chen W, Luo H, Chen W and Zhang H: Discrepant expression of microRNAs in transparent and cataractous human lenses. Invest Ophthalmol Vis Sci 53: 3906-3912, 2012.

19. Choi $\mathrm{J}$ and Donehower LA: p53 in embryonic development: Maintaining a fine balance. Cell Mol Life Sci 55: 38-47, 1999.

20. Lavker RM, Jia Yu and Ryan DG: The tiny world of microRNAs in the cross hairs of the mammalian eye. Hum Genomics 3 : 332-348, 2009.

21. Beebe DC, Holekamp NM and Shui YB: Oxidative damage and the prevention of age-related cataracts. Ophthalmic Res 44: $155-165,2010$

22. Brennan L, Khoury J and Kantorow M: Parkin elimination of mitochondria is important for maintenance of lens epithelial cel ROS levels and survival upon oxidative stress exposure. Biochim Biophys Acta 1863: 21-32, 2017.

23. Acer S, Pekel G, Kucukatay V, Küçükatay V, Karabulut A, Yağc1 R, Cetin EN, Akyer SP and Sahin B: Oxidative stress of crystalline lens in rat menopausal model. Arq Bras Oftalmol 79: 222-225, 2016.

24. Mohamed MF, Samir N, Ali A, Ahmed N, Ali Y, Aref S, Hossam O, Mohamed MS, Abdelmoniem AM and Abdelhamid IA: Apoptotic induction mediated p53 mechanism and Caspase-3 activity by novel promising cyanoacrylamide derivatives in breast carcinoma. Bioorg Chem 73: 43-52, 2017.
25. López-Luppo M, Catita J, Ramos D, Navarro M, Carretero A, Mendes-Jorge L, Muñoz-Cánoves P, Rodriguez-Baeza A, Nacher V and Ruberte J: Cellular senescence is associated with human retinal microaneurysm formation during aging. Invest Ophthalmol Vis Sci 58: 2832-2842, 2017.

26. Moshrefi M, Spotin A, Kafil HS, Mahami-Oskouei M, Baradaran B, Ahmadpour E and Mansoori B: Tumor suppressor p53 induces apoptosis of host lymphocytes experimentally infected by Leishmania major, by activation of Bax and caspase-3: A possible survival mechanism for the parasite. Parasitol Res 116: 2159-2166, 2017.

27. Mok JW, Chang DJ and Joo CK: Antiapoptotic effects of anthocyanin from the seed coat of black soybean against oxidative damage of human lens epithelial cell induced by $\mathrm{H} 2 \mathrm{O} 2$. Curr Eye Res 39: 1090-1098, 2014.

28. Zheng T and Lu Y: SIRT1 protects human lens epithelial cells against oxidative stress by inhibiting p53-dependent apoptosis. Curr Eye Res 41: 1068-1075, 2016.

29. Armand-Labit V and Pradines A: Circulating cell-free microRNAs as clinical cancer biomarkers. Biomol Concepts 8: 61-81, 2017.

30. Yang Y, Yu T, Jiang S, Zhang Y, Li M, Tang N, Ponnusamy M, Wang JX and Li PF: miRNAs as potential therapeutic targets and diagnostic biomarkers for cardiovascular disease with a particular focus on WO2010091204. Expert Opin Ther Pat 27: 1021-1029, 2017.

31. Wang S, Koster KM, He Y and Zhou Q: miRNAs as potential therapeutic targets for age-related macular degeneration. Future Med Chem 4: 277-287, 2012

32. Seto AG: The road toward microRNA therapeutics. Int J Biochem Cell Biol 42: 1298-1305, 2010.

33. Bruscella P, Bottini S, Baudesson C, Pawlotsky JM, Feray C and Trabucchi M: Viruses and miRNAs: More friends than foes. Front Microbiol 8: 824, 2017.

34. Ottosen S, Parsley TB, Yang L, Zeh K, van Doorn LJ, van der Veer E, Raney AK, Hodges MR and Patick AK: In vitro antiviral activity and preclinical and clinical resistance profile of miravirsen, a novel anti-hepatitis $\mathrm{C}$ virus therapeutic targeting the human factor miR-122. Antimicrob Agents Chemother 59: 599-608, 2015.

35. Gebert LF, Rebhan MA, Crivelli SE, Denzler R, Stoffel M and Hall J: Miravirsen (SPC3649) can inhibit the biogenesis of miR-122. Nucleic Acids Res 42: 609-621, 2014

36. Li X, Zhao F, Xin M, Li G, Luna C, Li G, Zhou Q, He Y, Yu B, Olson E, et al: Regulation of intraocular pressure by microRNA cluster miR-143/145. Sci Rep 7: 915, 2017.

37. SanGiovanni JP, SanGiovanni PM, Sapieha P and De Guire V: miRNAs, single nucleotide polymorphisms (SNPs) and age-related macular degeneration (AMD). Clin Chem Lab Med 55: 763-775, 2017.

38. Liu D, Sun X and Ye P: miR-31 overexpression exacerbates atherosclerosis by targeting NOX4 in apoE(-/-) mice. Clin Lab 61: $1617-1624,2015$.

39. Zhou Q, Gallagher R, Ufret-Vincenty R, Li X, Olson EN and Wang S: Regulation of angiogenesis and choroidal neovascularization by members of microRNA-23 27 24 clusters. Proc Natl Acad Sci USA 108: 8287-8292, 2011.

40. van Eijndhoven MA, Zijlstra JM, Groenewegen NJ, Drees EE, van Niele S, Baglio SR, Koppers-Lalic D, van der Voorn $H$, Libregts SF, Wauben MH, et al: Plasma vesicle miRNAs for therapy response monitoring in Hodgkin lymphoma patients. JCI Insight 1: e89631, 2016

41. Kang H, Rho JG, Kim C, Tak H, Lee H, Ji E, Ahn S, Shin AR, Cho HI, Huh YH, et al: The miR-24-3p/p130Cas: A novel axis regulating the migration and invasion of cancer cells. Sci Rep 7: 44847, 2017.

42. Cui S, Liao X, Ye C, Yin X, Liu M, Hong Y, Yu M, Liu Y, Liang H, Zhang CY and Chen X: ING5 suppresses breast cancer progression and is regulated by miR-24. Mol Cancer 16: 89, 2017.

43. Liu Z, Liu Z, Zhang Y, Li Y, Liu B and Zhang K: miR-24 represses metastasis of human osteosarcoma cells by targeting Ack1 via AKT/MMPs pathway. Biochem Biophys Res Commun 486: 211-217, 2017

44. Li YQ, Lu JH, Bao XM, Wang XF, Wu JH and Hong WQ: MiR-24 functions as a tumor suppressor in nasopharyngeal carcinoma through targeting FSCN1. J Exp Clin Cancer Res 34: 130, 2015.

45. Zhou Q, Anderson C, Zhang H, Li X, Inglis F, Jayagopal A and Wang S: Repression of choroidal neovascularization through actin cytoskeleton pathways by microRNA-24. Mol Ther 22 : 378-389, 2014.

This work is licensed under a Creative Commons Attribution-NonCommercial-NoDerivatives 4.0 International (CC BY-NC-ND 4.0) License. 